\title{
Use of Essential Oils and Plant Extracts to Control Microbial Contamination in Pet Food Products
}

\author{
Andreia Bianchini ${ }^{1,2 *}$, Jayne Stratton ${ }^{1,2}$, Steve Weier ${ }^{1}$, Carmen Cano² and Lucia Miceli Garcia ${ }^{2}$ \\ ${ }^{1}$ The Food Processing Center, 143 Food Industry Complex, University of Nebraska - Lincoln, Lincoln NE USA \\ ${ }^{2}$ Department of Food Science and Technology, 143 Food Industry Complex, University of Nebraska - Lincoln, Lincoln NE USA
}

\begin{abstract}
The antimicrobial activity of 25 essential oils and plant extracts against Salmonella Typhimurium and Penicillium roqueforti was tested by disc diffusion and quantified by agar dilution. Afterwards, the effect of the most promising essential oils was studied in an extruded pet food product over 21 days, with the oil mixed either into the product or as part of its coating. In vitro, the best inhibitors for Salmonella were cinnamon essential oil (EO) at $0.05 \%$ and thyme EO at $0.1 \%$, while P. roqueforti was best inhibited by cinnamon EO at $0.01 \%$ and spearmint EO at $0.5 \%$. When tested in the extruded product, cinnamon EO $(0.05 \%$ and $0.1 \%)$ and spearmint EO $(0.5 \%)$ proved ineffective against $P$. roqueforti, either mixed into the product or as part of its coating. Only cinnamon EO $(0.1 \%)$ as part of the coating was able to reduce Salmonella significantly faster than the control $(p$-value $=0.0408)$ during storage for 21 days. Based on the results, spice essential oils can act as inhibitors of Salmonella in a pet food product, when present at a sufficient concentration.
\end{abstract}

Keywords: Pet food products; Salmonella Typhimurium; Penicillium roqueforti; Essential oils; Natural antimicrobials

\section{Introduction}

The Centers for Disease Control and Prevention (CDC) has reported more than 19,000 laboratory-confirmed cases of foodborne infection for 2012, with Salmonella as the cause of more than $40 \%$ of the cases (Centers for Disease Control and Prevention (CDC), 2013) [1]. Recently, outbreaks of human salmonellosis have been associated with dried pet foods, and pet treats containing beef, dried beef, seafood, or pig ears; the most recent one occurred in 2012 [1-5]. The CDC has associated the human infections with handling of the contaminated product [2].

Another problem faced by the pet food industry relates to economic losses due to mold spoilage. The actual economic value of this problem is difficult to quantify because the industry does not report numbers on the issue. However, this is certainly of interest to the pet food industry, especially with the new generation of products that have soft and hard pieces in the same bag. The higher moisture content of the product increases palatability, but makes it necessary to add humectants or organic acids to control water activity [6]

In recent years increased public concern over chemical feed and food additives has prompted searches for more natural antimicrobial agents. One source of such agents are essential oils, spices and plant extracts that could be used in pet food products as a natural alternative to control microbial contamination. Accomplishing this would benefit the pet food industry by improving product safety and quality; and most importantly protect pet owners from the hazard associated with handling potentially contaminated products.

In vitro experiments have demonstrated the inhibitory activity of different essential oils against spoilage molds and pathogenic bacteria. Hammer et al. [7] showed the effectiveness of plant oils and extracts from rosemary, oregano, lemongrass, sage, clove, and thyme against Salmonella Typhimurium, with Minimum Inhibitory Concentration (MIC) ranging from $0.12 \%$ to more than $2.0 \%$. Smith-Palmer et al. [8] evaluated the effects of 21 essential oils against five foodborne pathogens, including Salmonella Enteritidis, and oils of bay, cinnamon, clove and thyme presented the higher inhibitory activity with concentrations of $0.075 \%$ or lezzouz and Bullerman [9]. A described the inhibitory effect of cloves, cinnamon, mustard, allspice, garlic, and oregano against seven mycotoxin-producing molds; while Paster et al. [10] showed the inhibitory effect of oregano and thyme oil on the growth of Aspergillus $s p p$. and natural microflora of wheat. Some essential oils, such as mint and oregano, have also been reported as active against Salmonella in food systems, which are a more complex environment than laboratory culture media [11-13].

The addition of essential oils to pet food products or animal feed has been suggested as breath-freshening agents [14], palatability enhancers $[15,16]$ or antimicrobials $[17,18]$ in different patents. To this date, however, there have been no challenge studies to support the claim of inhibitory activity in an extruded pet food product. This study aimed to evaluate the effect of essential oils and plant extracts in controlling microbial contamination in extruded pet food products, by testing them in vitro against organisms of interest, determining their Minimum Inhibitory Concentration (MIC) and then incorporating them into pet food products for challenge studies.

\section{Materials and Methods}

\section{Testing of essential oils and plant extracts}

Based on a literature review, essential oils and alcohol-based plant extracts with reported antimicrobial activity (Table 1) were selected for testing against Salmonella Typhimurium ATCC 14028 and Penicillium roqueforti NRRL 849 by disc diffusion assay. Salmonella enterica subsp. enterica Typhimurium is one of the two most common serovars linked to human salmonellosis, while $P$. roqueforti is a common spoilage fungi for animal feed $[19,20]$.

*Corresonding author: Andreia Bianchini, Department of Food Science and Technology, 143 Food Industry Complex, University of Nebraska - Lincoln, Lincoln NE USA, Tel: +1 402-472-3114; Fax: +1 402-472-1693; E-mail: abianchini2@unl.edu

Received Aug 12, 2014; Accepted Sep 15, 2014; Published Sep 23, 2014

Citation: Bianchini A, Stratton J, Weier S, Cano C, Garcia LM et al. (2014) Use of Essential Oils and Plant Extracts to Control Microbial Contamination in Pet Food Products. J Food Process Technol 5: 357. doi:10.4172/2157-7110.1000357

Copyright: @ 2014 Bianchini A, et al. This is an open-access article distributed under the terms of the Creative Commons Attribution License, which permits unrestricted use, distribution, and reproduction in any medium, provided the original author and source are credited. 


\begin{tabular}{|c|c|c|}
\hline Common Name & Scientific Name & Source \\
\hline \multicolumn{3}{|l|}{ Alcohol-based Extracts } \\
\hline Anise & Illicium verum & Tone's/ACH Food Companies, Inc \\
\hline Lemon & Citrus limon & Green Mountain Flavors, Inc. \\
\hline Lime & Citrus aurantifolia & Green Mountain Flavors, Inc. \\
\hline Cinnamon & Cinnamomum cassia & Green Mountain Flavors, Inc. \\
\hline Peppermint & Mentha piperita & McCormick \& Co., Inc. \\
\hline \multicolumn{3}{|l|}{ Essential Oils } \\
\hline Tea tree & Malaleuca alternifolia & Green Valley Aromatherapy Ltd. \\
\hline Eucalyptus citridora & Eucalyptus citridora & Green Valley Aromatherapy Ltd. \\
\hline Eucalyptus globulus & Eucalyptus globulus & Green Valley Aromatherapy Ltd. \\
\hline Lime & Citrus aurantifolia & Green Valley Aromatherapy Ltd. \\
\hline Sandalwood Aus & Santalum spicatum & Green Valley Aromatherapy Ltd. \\
\hline Melissa (true) & Melissa officianalis & Green Valley Aromatherapy Ltd. \\
\hline Basil sweet & Ocimum basilicum & Starwest Botanicals, Inc. \\
\hline Peppermint & Mentha piperita & Starwest Botanicals, Inc. \\
\hline Black pepper & Piper nigrum & Starwest Botanicals, Inc. \\
\hline Anise & Illicium verum & Starwest Botanicals, Inc. \\
\hline Ginger & Zingiber officinale & Starwest Botanicals, Inc. \\
\hline Clary sage & Salvia sclarea & Starwest Botanicals, Inc. \\
\hline Cinnamon leaf & Cinnamomum zeylanicum & Starwest Botanicals, Inc. \\
\hline
\end{tabular}

Table 1: Spices, essential oils and extracts selected for testing of antimicrobial activity.

The inhibitory activity of the chosen essential oils and plant extracts was tested in a disc diffusion assay, using Salmonella enterica subsp. enterica Typhimurium ATCC 14028 and Penicillium roqueforti NRRL 849. For testing inhibition against Salmonella, tryptic soy agar (TSA; Acumedia, Neogen Corporation, Lansing, MI) was inoculated with $1 \%(\mathrm{v} / \mathrm{v})$ of an overnight culture grown in tryptic soy broth (TSB; Acumedia, Neogen Corporation, Lansing, MI) at $37 \pm 2{ }^{\circ} \mathrm{C}$, to achieve an approximate concentration of 106 $\mathrm{CFU} / \mathrm{ml}$ of TSA. For testing inhibition against $P$. roqueforti, potato dextrose agar (PDA, Acumedia, Neogen Corporation, Lansing, $\mathrm{MI})$ was inoculated with sufficient volume of a spore suspension to achieve a concentration of $1 \times 10^{4}$ spores $/ \mathrm{ml}$ of PDA. Agar plates were prepared with $25 \mathrm{ml}$ of inoculated media in each and left to dry for 30 minutes. Then, three sterilized paper discs $(3 \mathrm{~mm}$ in diameter, made from Whatman filter paper no. 3) containing $5 \mu \mathrm{L}$ of essential oil or plant extract were placed on each plate. Salmonella plates were incubated at $37 \pm 2^{\circ} \mathrm{C}$ for 24 hours and $P$. roqueforti plates were incubated at $25 \pm 2{ }^{\circ} \mathrm{C}$ for 5 days. After incubation, the diameter of the inhibition zones around each disc was measured using a colony counter (Flash and Grow, IUL Instruments, Neutec Group Inc, Farmingdale, NY). Three replicates were prepared for each extract and oil tested. The inhibition activity was classified as none $($ diam $<8$ $\mathrm{mm})$; slight $(8 \mathrm{~mm}<$ diam $<11 \mathrm{~mm})$; moderate $(11 \mathrm{~mm}<$ diam $<16$ $\mathrm{mm}$ ); or strong (diam $>16 \mathrm{~mm})$. Alcohol $90 \%$ was used as a control for alcoholic plant extracts. Its activity was subtracted from that of the plant extracts. Those extracts and oils with greater inhibition zones for each microorganism were selected for the following phase, after reviewing the literature for their suitability for animal consumption.
Testing of combinations and minimum inhibitory concentra tions of essential oils

In an attempt to find a combination that could further inhibit both Salmonella and $P$. roqueforti, the diffusion disc assay was repeated with combinations of the essential oils chosen in the previous phase. The oils were mixed in a 1:1 proportion. Plant extracts did not show a high inhibitory activity and were discarded for this phase. Analysis of variance was used to compare the inhibition zones of oil combinations against the inhibitions zones of cinnamon, the essential oil with highest activity. The analysis was conducted on zone diameters, with type of essential oil as the independent variable. Statistical analysis was carried out using SAS 9.3 (SAS, Inc, Cary, NC) and all tests were conducted at the $5 \%$ level of significance.

After the best essential oils and combinations were chosen, the Minimum Inhibitory Concentration (MIC) for each one was determined. To accomplish this, the oils or combinations were added in a range of concentrations ( 0.0 to $1.0 \% \mathrm{v} / \mathrm{v})$ to inoculated agar in 12-well plates. The agar used for the 12-well plates were inoculated in the same manner as described for the disc diffusion assay. However, Salmonella inoculation level was raised to $5 \%(\mathrm{v} / \mathrm{v})$, or about $5 \times 10^{6} \mathrm{CFU} / \mathrm{ml} \mathrm{TSA}$, for ease of result reading. After incubation, microbial growth in the wells containing essential oil or a combination of essential oils was compared to the microbial growth obtained in the control wells $(0.0 \%$ $\mathrm{v} / \mathrm{v}$ oil). The MIC was defined as the lowest concentration of essential oil needed to visibly inhibit mold or bacterial growth.

\section{Incorporation of oils into pet food and challenge studies}

An extruded pet food product was prepared, following the recipe 
on Table 2, to achieve water activity around 0.8. Essential oils were incorporated as part of the recipe of the uncoated product. Another product with no essential oil added was prepared, to serve as control for the uncoated product challenge study and to be coated, with or without addition of essential oils into the coating recipe. The oils used for the Salmonella challenge study were cinnamon (0.05\% and $0.1 \%)$ and thyme $(0.1 \%)$; while the oils used for the $P$. roqueforti challenge study were cinnamon $(0.05 \%$ and $0.1 \%)$ and spearmint $(0.5 \%)$. Two batches of pet food product were prepared for coated and uncoated product in independent extrusions. The water activity of the products was measured using an Aqualab Model Series 3 water activity meter (Decagon Devices Inc, Pullman, WA).

The pet food formulation consisted of a dry base and a slurry (Table 2 ), which were prepared separately and then mixed in a countertop mixer (Legacy countertop mixer, Hobart, Troy, $\mathrm{OH}$ ) for roughly 3 minutes. The prepared product mix was extruded in a conical twin screw extruder with single mixing zone screws and a $7 \mathrm{~mm} \mathrm{ID}$ nozzle insert (Co-Rotating Twin Screw Extruder, C. W. Brabender Instruments, South Hackensack, NJ). The configuration of the screw inside the extruder included a diameter of $31.1 \mathrm{~mm}$ at the inlet end and a total screw length of $330.7 \mathrm{~mm}$. Temperature settings at the different barrel zones were $50^{\circ} \mathrm{C}-100^{\circ} \mathrm{C}-110^{\circ} \mathrm{C}-110^{\circ} \mathrm{C}$, with a screw speed of $120 \mathrm{rpm}$. The prepared mix was metered by hand into the extruder due to its stickiness. The extruded pieces were cut upon exiting the die using a rotary knife resulting in a piece size of $9 \mathrm{~mm}$ dia $\mathrm{x} 9 \mathrm{~mm}$ in length.

For each treatment, 25-gram samples were placed aseptically in a sterile filter bag (Whirl-Pak, Nasco, Ft. Atkinson, WI) and enough inoculum was added to achieve the desired levels of either Salmonella $\left(10^{5} \mathrm{CFU} / \mathrm{g}\right.$; and $\left.10^{7} \mathrm{CFU} / \mathrm{g}\right)$ or P. roqueforti $\left(10^{3} \mathrm{spores} / \mathrm{g}\right)$. Controls of each product (uncoated and coated) with no essential oil added were inoculated as well. For this challenge study, 8 bags were prepared from each extrusion, totaling 16 bags for each combination of product treatment, organism and inoculation level.

Products were stored at room temperature and sampled at days $1,7,14$ and 21 to determine the presence and levels of Salmonella Typhimurium or P. roqueforti. To enumerate the microorganisms in the samples, serial dilutions were made using Phosphate Buffered Saline (PBS; Sigma, St. Louis, MO) to achieve a 1:10 dilution and samples were plated at appropriate dilutions. The presence of Salmonella was determined by plating on Xylose Lactose Tergitol ${ }^{\mathrm{mw}} 4$ agar (XLT4; Acumedia, Neogen Corporation, Lansing, MI) and incubating for 24 hours at $37 \pm 2{ }^{\circ} \mathrm{C}$. The presence of $P$. roqueforti was determined by plating on PDA and incubating for 5 days at $25 \pm 2^{\circ} \mathrm{C}$. Both enumeration methods had a level of detection of less than $10 \mathrm{CFU} / \mathrm{g}$ of product.

Analysis of variance was used to evaluate the effect of type and concentration of essential oil on the behavior of microorganisms. The microbial counts for each day were expressed in logarithmic form and used to prepare a linear regression, from which a growth/death rate (CFU/g per day) was obtained. The analysis was conducted on $P$. roqueforti growth rates or $\mathrm{S}$. Typhimurium death rates with essential oil treatment as the independent variable. Statistical analysis was carried out using SAS 9.3 (SAS, Inc, Cary, NC) and all tests were conducted at the $5 \%$ level of significance. Tukey's post-comparison method was used to evaluate significant differences among the means.

\section{Results}

\section{Testing of essential oils and plant extracts}

Table 3 shows the results for the inhibitory activity of essential oils and plant extracts, classified according to the diameter of the inhibition halos. Even though tea tree, melissa and black spruce oils showed inhibitory activity varying from slight to strong, they were not evaluated further because there was not enough evidence in the literature to

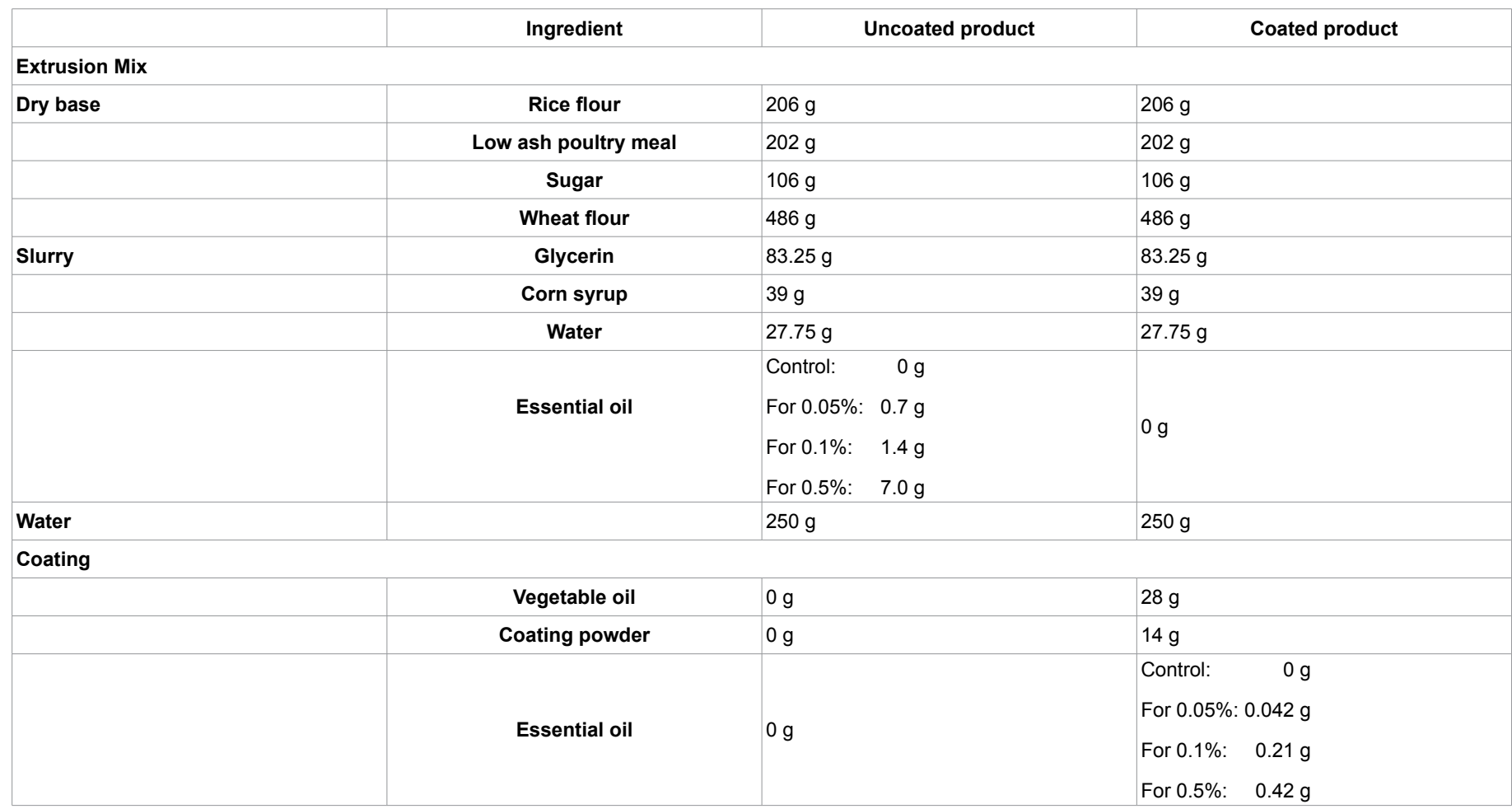

Table 2: Recipe for uncoated and coated extruded pet food products ( $\% \mathrm{w} / \mathrm{w}$ ) 


\begin{tabular}{|c|c|c|}
\hline & Inhibition of Salmonella classification ${ }^{a}$ & Inhibition of $P$. roqueforti classification \\
\hline \multicolumn{3}{|l|}{ Essential Oils } \\
\hline Tea tree & Moderate & Moderate \\
\hline Eucalyptus citridora & None & Strong \\
\hline Eucalyptus globulus & Slight & None \\
\hline Lime & Moderate & Moderate \\
\hline Black spruce & Slight & Strong \\
\hline Benchmark thyme & Moderate & Strong \\
\hline Manuka & None & None \\
\hline Patchouli & None & None \\
\hline Sandalwood & None & None \\
\hline Melissa (true) & Slight & Strong \\
\hline Sweet basil & Slight & None \\
\hline Peppermint & None & Strong \\
\hline Black pepper & None & None \\
\hline Anise & None & Not available \\
\hline Ginger & None & None \\
\hline Clary sage & None & Moderate \\
\hline Cinnamon leaf & Moderate & Strong \\
\hline Cinnamon & Strong & Strong \\
\hline Spearmint & Moderate & Strong \\
\hline Lemon & None & None \\
\hline \multicolumn{3}{|l|}{ Plant Extracts } \\
\hline Anise & Slight & None \\
\hline Lemon & Moderate & None \\
\hline Lime & Slight & Not available \\
\hline Cinnamon & Moderate & None \\
\hline Peppermint & Slight & Slight \\
\hline Mint & Moderate & None \\
\hline Alcohol $90 \%$ & Slight & Slight \\
\hline
\end{tabular}

aClassification for inhibition: none (diam<8 mm); slight ( $8 \mathrm{~mm}<$ diam<11 mm); moderate $(11 \mathrm{~mm}<$ diam<16 mm); strong (diam>16mm).

Table 3: Inhibitory activity of essential oils and plant extracts against Salmonella and $P$. roqueforti

support their safety for animal consumption. The essential oils with the highest antimicrobial activity were lime, thyme and cinnamon for Salmonella; and eucalyptus citridora, thyme, peppermint, cinnamon and spearmint for P. roqueforti. Because of their antimicrobial activity, these oils were selected for testing against both organisms of interest in combination experiments (ratio 1:1) to evaluate for a potential synergistic effect.

\section{Testing of combinations and minimum inhibitory concentra tions of essential oils}

Table 4 shows the results on the inhibitory activity of combinations of essential oils, as determined by the disc assays. According to the size of the inhibition zones, the best combination for inhibiting both Salmonella and $P$. roqueforti was cinnamon-spearmint, while cinnamon-thyme was also good for inhibiting Salmonella and cinnamon-peppermint proved effective against $P$. roqueforti.

Based on the results shown on Tables 3 and 4, cinnamon, thyme, spearmint, peppermint and eucalyptus essential oils and the combinations cinnamon-spearmint, cinnamon-thyme and cinnamonpeppermint were used to determine the MIC in the 12-well plate assay. The results are presented in Table 5.

Based on the MIC, cinnamon was chosen to be tested in the challenge studies as an inhibitor of both Salmonella and $P$. roqueforti. However, because it was expected that the effectiveness in the pet food product would be lower than that observed in vitro, different concentrations were included $(0.05 \%$ and $0.1 \%)$. Thyme was chosen because of its promising activity as an inhibitor of Salmonella and spearmint as an inhibitor of $P$. roqueforti. The combinations were not used, since the results did not show a significant improvement over the individual oils when the MIC was determined.

\section{Incorporation of oils into pet food and challenge studies}

Figures 1 and 2 show the reduction of Salmonella and the growth of $P$. roqueforti in the extruded pet food, respectively. When the death rates for Salmonella and the growth rates for $P$. roqueforti were calculated and evaluated by statistical analysis, none of the products with essential oil inoculated with mold showed a significantly different growth rate from that of the controls (Table 6). For products inoculated with Salmonella (Table 7), no significant differences were present amongst the treatments in which the oils were added directly into the product or in those products inoculated with $10^{7} \mathrm{CFU} / \mathrm{g}$. In the coated products inoculated with $10^{5} \mathrm{CFU} / \mathrm{g}$, the addition of $0.1 \%(\mathrm{w} / \mathrm{w})$ cinnamon essential oil into the coating exhibited a death rate which was significantly different from that of the control ( $p$-value $=0.0431$, but not from the other treatments $(0.05 \%$ cinnamon $\mathrm{p}$-value $=0.7148$, $0.1 \%$ thyme $\mathrm{p}$-value $=0.0506$ ). Therefore, this treatment may be effective in the inhibition of Salmonella in an extruded pet food product.

\section{Discussion}

The microbial inhibitory activity of alcohol plant extracts and essential oils against Salmonella and P. roqueforti was tested, both in vitro and in a pet food product. Salmonella enterica subsp. enterica serovar Typhimurium has been the second most common cause of 


\begin{tabular}{|c|c|c|c|c|}
\hline \multirow[b]{2}{*}{ Combination } & \multicolumn{2}{|c|}{ Inhibition of Salmonella } & \multicolumn{2}{|c|}{ Inhibition of $P$. roqueforti } \\
\hline & Inhibition zone (diameter, $\mathrm{mm})^{\mathrm{a}}$ & Classification $^{\mathrm{b}}$ & Inhibition zone (diameter, $\mathrm{mm})^{\mathrm{a}}$ & Classification $^{\mathrm{b}}$ \\
\hline Cinnamon & $20.66 \pm 4.45^{\mathrm{a}, \mathrm{b}}$ & Strong & $25.4 \pm 4.48)^{c}$ & Strong \\
\hline Cinnamon-Spearmint & $21.14 \pm 4.37^{\mathrm{a}}$ & Strong & $40.21 \pm 4.61)^{\mathrm{a}}$ & Strong \\
\hline Cinnamon-Thyme & $20.90 \pm 3.15^{\mathrm{a}, \mathrm{b}}$ & Strong & $34.78 \pm 3.38)^{a, b}$ & Strong \\
\hline Cinnamon-Eucalyptus & $19.83 \pm 1.36^{a, b}$ & Strong & $33.11 \pm 2.54)^{b}$ & Strong \\
\hline Cinnamon-Peppermint & $16.60 \pm 0.19^{a, b, c}$ & Moderate & $40.22 \pm 1.53)^{\mathrm{a}}$ & Strong \\
\hline Lime-Cinnamon & $18.95 \pm 0.71^{\mathrm{a}, \mathrm{b}}$ & Moderate & $38.15 \pm 1.41)^{\mathrm{a}, \mathrm{b}}$ & Strong \\
\hline Lime-Spearmint & $11.37 \pm 0.84^{\mathrm{c}, \mathrm{d}}$ & Slight & $8.95 \pm 0.37)^{\mathrm{e}}$ & Slight \\
\hline Lime-Thyme & $13.71 \pm 2.45 b^{\mathrm{ccd} d}$ & Moderate & $9.77 \pm 1.18)^{\mathrm{e}}$ & Slight \\
\hline Lime-Eucalyptus & $9.12 \pm 1.35^{d}$ & Slight & $7.03 \pm 0.20)^{\mathrm{e}}$ & None \\
\hline Lime-Peppermint & $9.53 \pm 1.04^{d}$ & Slight & $7.00 \pm 0.00)^{\mathrm{e}}$ & None \\
\hline Thyme-Spearmint & $8.58 \pm 0.70^{d}$ & Slight & $16.96 \pm 0.96)^{d}$ & Moderate \\
\hline Thyme-Eucalyptus & $9.27 \pm 1.04^{d}$ & Slight & $10.09 \pm 1.31)^{\mathrm{e}}$ & Slight \\
\hline Thyme-Peppermint & $9.55 \pm 0.89^{d}$ & Slight & $10.98 \pm 0.70)^{\mathrm{e}}$ & Slight \\
\hline Spearmint-Eucalyptus & $7.00 \pm 0.00^{d}$ & None & $9.88 \pm 0.84)^{e}$ & Slight \\
\hline Spearmint-Peppermint & $8.58 \pm 0.34^{d}$ & Slight & $9.57 \pm 0.72)^{e}$ & Slight \\
\hline
\end{tabular}

a Inhibition zone diameters are average \pm standard deviation) of three measurements. Diameters with a different letter are significantly different ( $<<0.05)$ from each other. ${ }^{b}$ Classification for inhibition: none (diam< $8 \mathrm{~mm}$ ); slight $(8 \mathrm{~mm}<\operatorname{diam}<11 \mathrm{~mm})$; moderate $(11 \mathrm{~mm}<\operatorname{diam}<16 \mathrm{~mm})$; strong $($ diam $>16 \mathrm{~mm})$.

Table 4: Inhibitory activity of 1:1 combinations of essential oils against Salmonella Typhimurium and Penicillium roqueforti

\begin{tabular}{|c|c|c|}
\hline Essential oil or Combination & $\begin{array}{c}\text { MIC for Salmonella } \\
(\% \mathbf{q} / \mathbf{v})\end{array}$ & $\begin{array}{c}\text { MIC for P. roqueforti } \\
(\% \mathbf{v} / \mathbf{v})\end{array}$ \\
\hline Cinnamon & 0.05 & 0.01 \\
\hline Thyme & 0.1 & N.T. \\
\hline Spearmint & $>1.0$ & 0.5 \\
\hline Eucalyptus & N.T.a & 1.0 \\
\hline Peppermint & N.T. & $>1.0$ \\
\hline Cinnamon-Spearmint & 0.05 & 0.01 \\
\hline Cinnamon-Thyme & 0.05 & N. T. \\
\hline Cinnamon-Peppermint & N.T. & 0.01 \\
\hline
\end{tabular}

aN. T.: Not Tested

Table 5: Minimum inhibitory concentration (MIC, \%v/v) of essential oils against Salmonella Typhimurium and Penicillium roqueforti

salmonellosis outbreaks in the United States for the last three years [20]. On the other hand, P. roqueforti is a common spoilage fungi in animal feed and it is capable of producing mycotoxins related to animal health disorders [19]. In the screening phase of this research, essential oils showed inhibitory activity varying from slight to strong against the microorganisms tested, while alcohol extracts showed little or no activity. The method of extraction and storage, along with harvesting factors, has been shown to have an effect on antimicrobial activity of essential oils [21].

In this assay, cinnamon, thyme and lime essential oils showed the greater activity against Salmonella, while eucalyptus citriodora, thyme, peppermint, cinnamon and spearmint showed strong activity against $P$. roqueforti. The activity observed was consistent with that reported in the literature. Cinnamon and thyme are considered among the spices and herbs with stronger antimicrobial activity, while peppermint and spearmint have been reported to show limited activity [22]. Phenolic components are thought to be responsible for this antimicrobial activity. Cinnamon contains both eugenol (2-methoxy-4-allyl phenol) and cinnamic aldehyde, which are also present in allspice and cloves [23]. The antimicrobial activity of thyme, on the other hand, is attributed to the terpenes carvacrol, p-cymene, and thymol [23]. Isoeugenol, cinnamaldehyde, carvacrol and thymol have shown strong antifungal activity as well, as described by Suhr and Nielsen [24] when testing cinnamon and thyme oils against four spoilage fungi, including P. roqueforti. Prabuseenivasan et al. [25] found that essential oil extracted from lime showed significant activity against Staphylococcus aureus, Bacillus subtilis, Klebsiella pneumoniae, Proteus vulgaris, Pseudomonas aeruginosa and Escherichia coli, though cinnamon showed higher activity. The same researchers showed that eucalyptus citridora essential oil had no inhibitory effect on these bacteria [25]. However, Alfazairy [26] described its strong activity against Penicillium spp. Mint and peppermint essential oils have also been reported as having intermediate activity against Gram-negative pathogenic bacteria and eleven fungi $[27,28]$.

After selecting the essential oils with the most promising antimicrobial activity against Salmonella Typhimurium and $P$. roqueforti, the effect of their combination and concentration was tested. Delaquis et al. [29] achieved a synergistic effect when combining fractions of eucalyptus and cilantro essential oils against Yersinia enterocolitica and additive effects against Gram-positive bacteria and Saccharomyces cerevisiae. In this research, combinations of the chosen oils were tested to determine if an additive or synergistic effect could be achieved against one or both of the microorganisms. A synergistic effect would make it possible to decrease the concentration of essential oil and reduce its impact on 


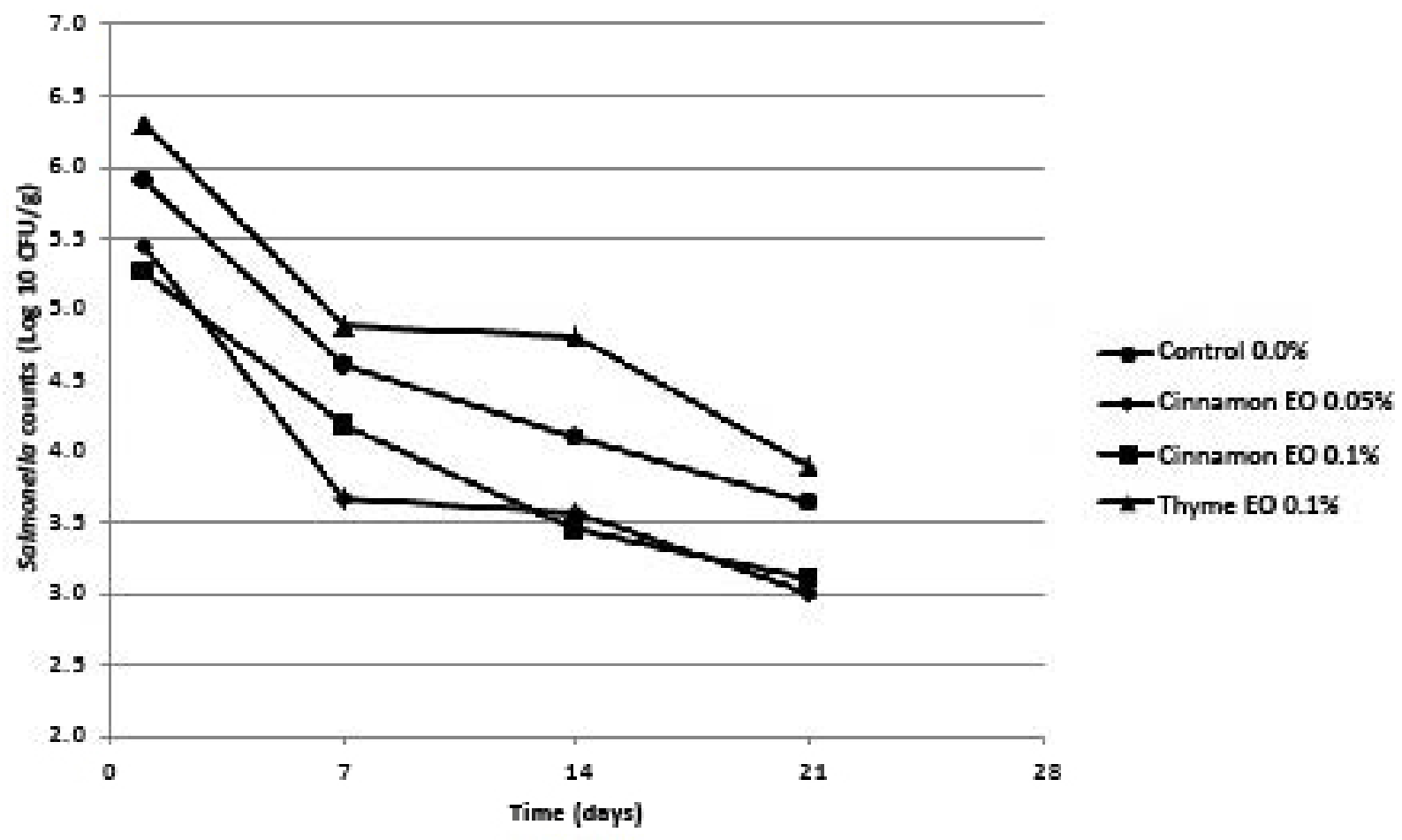

Figure 1: Salmonella reduction in extruded pet food product with essential oil as part of the product and high initial inoculation (estimated $10^{7} \mathrm{cfu} / \mathrm{g}$ ).

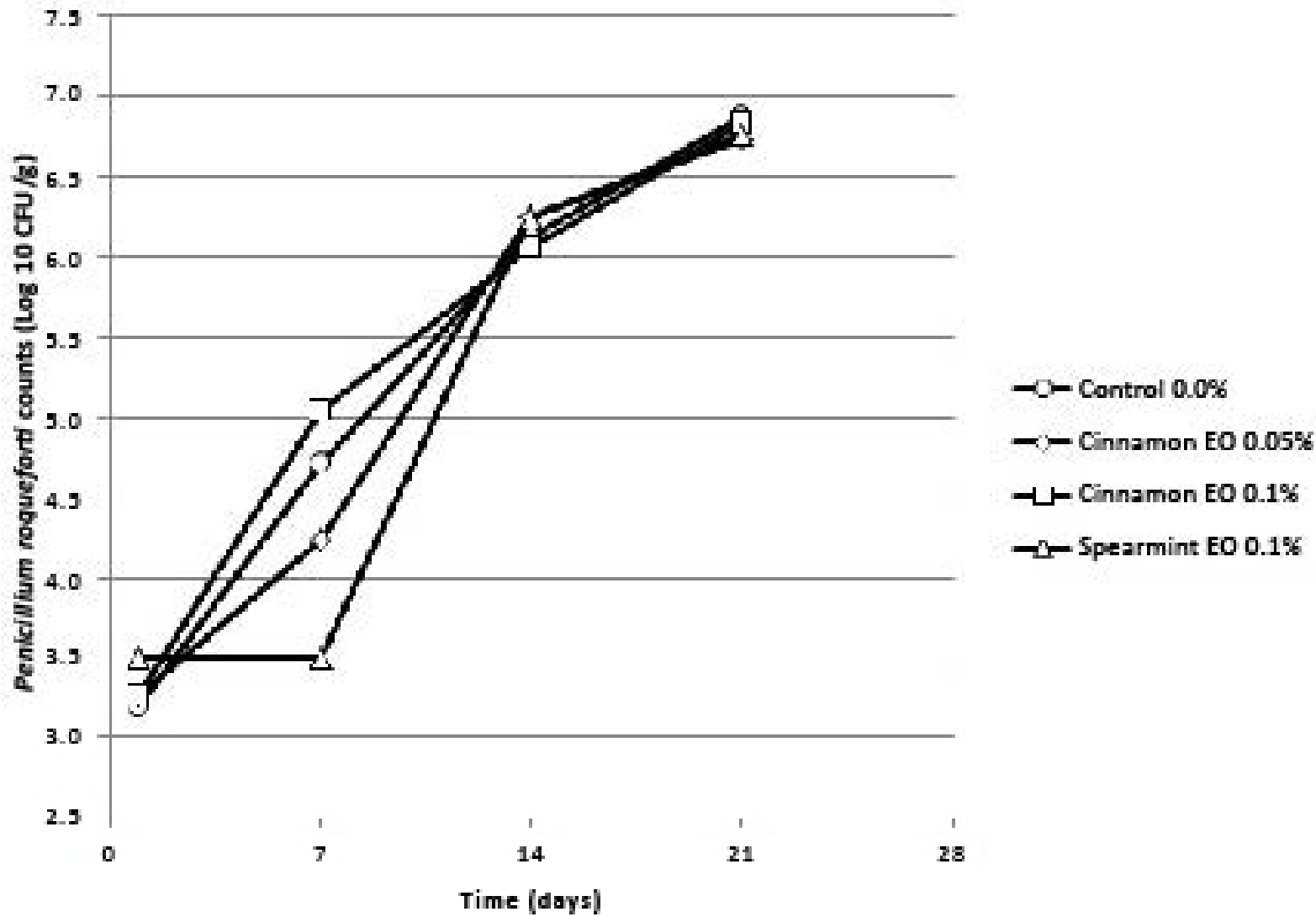

Figure 2: Penicillium roqueforti growth in extruded pet food with essential oil as part of the coating. 


\begin{tabular}{|c|c|c|c|c|c|c|c|}
\hline Type of Addition & Essential Oil & $\begin{array}{l}\text { Day } 0 \text { Count } \\
(\log 10 \mathrm{CFU} / \mathrm{g})\end{array}$ & $\begin{array}{l}\text { Day } 7 \text { Count } \\
(\log 10 \text { CFU/g) }\end{array}$ & $\begin{array}{l}\text { Day } 14 \text { Count } \\
(\log 10 \mathrm{CFU} / \mathrm{g})\end{array}$ & $\begin{array}{l}\text { Day } 21 \text { Count } \\
\text { (log10 CFU/g) }\end{array}$ & $\begin{array}{c}\text { Total Growth } \\
(\Delta \log 10 \mathrm{CFU} / \mathrm{g})\end{array}$ & $\begin{array}{l}\text { Increase in counts/day } \\
(\Delta \log 10 \mathrm{CFU} / \mathrm{g} \text { per day })^{\mathrm{a}}\end{array}$ \\
\hline Product & Control $(0.0 \%)$ & 3.3 & 3.3 & N.A.b & 6.7 & 3.50 & $0.173 a$ \\
\hline Product & Cinnamon $(0.05 \%)$ & 3.2 & 3.2 & N.A. & 6.6 & 3.40 & 0.169 a \\
\hline Product & Cinnamon $(0.1 \%)$ & 3.1 & 3.1 & N.A. & 6.9 & 3.80 & $0.192 \mathrm{a}$ \\
\hline Product & Spearmint $(0.5 \%)$ & 3.0 & 3.0 & N.A. & 6.0 & 3.00 & $0.151 \mathrm{a}$ \\
\hline Coating & Control $(0.0 \%)$ & 3.2 & 3.2 & 6.2 & 6.9 & 3.70 & $0.211 \mathrm{a}$ \\
\hline Coating & Cinnamon $(0.05 \%)$ & 3.3 & 3.3 & 6.3 & 6.7 & 3.40 & 0.199 a \\
\hline Coating & Cinnamon $(0.1 \%)$ & 3.3 & 3.4 & 6.1 & 6.8 & 3.60 & $0.202 \mathrm{a}$ \\
\hline Coating & Spearmint $(0.5 \%)$ & 3.4 & 3.4 & 6.3 & 6.5 & 3.20 & $0.185 \mathrm{a}$ \\
\hline
\end{tabular}

ancrease in counts per day with a different letter are significantly different $(p<0.05)$ from rates for the same type of addition. ${ }^{b}$ N.A.: Not Available.

Table 6: Increase in counts of Penicillium roqueforti in extruded pet food products

\begin{tabular}{|c|c|c|c|c|c|c|c|}
\hline Type of Addition & Essential Oil & $\begin{array}{c}\text { Day } 0 \text { Count } \\
\text { (log10 CFU/g) }\end{array}$ & $\begin{array}{c}\text { Day } 7 \text { Count } \\
\text { (log10 CFU/g) }\end{array}$ & $\begin{array}{l}\text { Day } 14 \text { Count } \\
(\log 10 \mathrm{CFU} / \mathrm{g})\end{array}$ & $\begin{array}{l}\text { Day } 21 \text { Count } \\
(\log 10 \text { CFU/g) }\end{array}$ & $\begin{array}{l}\text { Total Reduction } \\
(\Delta \log 10 \mathrm{CFU} / \mathrm{g})\end{array}$ & $\begin{array}{c}\text { Reduction/day } \\
(\Delta \log 10 \mathrm{CFU} / \mathrm{g} \\
\text { per day })^{\mathrm{a}}\end{array}$ \\
\hline \multicolumn{8}{|c|}{ Inoculation level - Low } \\
\hline Product & Control $(0.0 \%)$ & 2.5 & 2.2 & 0.8 & 0.7 & 1.8 & $0.102 \mathrm{a}$ \\
\hline Product & Cinnamon $(0.05 \%)$ & 2.8 & 2.8 & 1.3 & 0.0 & 2.8 & 0.148 a \\
\hline Product & Cinnamon $(0.1 \%)$ & 2.1 & 2.1 & 0.0 & 0.0 & 2.1 & $0.125 \mathrm{a}$ \\
\hline Product & Thyme $(0.1 \%)$ & 2.9 & 2.9 & 0.0 & 0.0 & 2.3 & $0.145 \mathrm{a}$ \\
\hline Coating & Control $(0.0 \%)$ & 3.3 & 2.7 & 1.8 & 0.0 & 3.3 & $0.172 \mathrm{a}$ \\
\hline Coating & Cinnamon $(0.05 \%)$ & 4.5 & 4.5 & 1.8 & 0.0 & 4.5 & $0.244 a, b$ \\
\hline Coating & Cinnamon $(0.1 \%)$ & 4.4 & 4.4 & 0.7 & 0.0 & 4.4 & $0.255 \mathrm{~b}$ \\
\hline Coating & Thyme $(0.1 \%)$ & 3.1 & 3.1 & 0.7 & 0.0 & 3.1 & $0.177 a, b$ \\
\hline \multicolumn{8}{|c|}{ Inoculation level - High } \\
\hline Product & Control $(0.0 \%)$ & 5.9 & 5.9 & 2.8 & 3.6 & 2.3 & $0.151 \mathrm{a}$ \\
\hline Product & Cinnamon $(0.05 \%)$ & 5.5 & 5.5 & 3.5 & 2.7 & 2.7 & $0.151 \mathrm{a}$ \\
\hline Product & Cinnamon $(0.1 \%)$ & 5.2 & 5.2 & 3.2 & 2.9 & 2.3 & $0.135 \mathrm{a}$ \\
\hline Product & Thyme $(0.1 \%)$ & 6.3 & 6.3 & 4.8 & 3.4 & 2.9 & $0.157 \mathrm{a}$ \\
\hline Coating & Control $(0.0 \%)$ & 6.6 & 6.6 & 3.9 & 5.0 & 1.6 & $0.110 \mathrm{a}$ \\
\hline Coating & Cinnamon $(0.05 \%)$ & 6.4 & 6.4 & 5.2 & 2.7 & 3.7 & $0.184 \mathrm{a}$ \\
\hline Coating & Cinnamon $(0.1 \%)$ & 6.4 & 6.4 & 4.7 & 4.5 & 1.9 & $0.110 \mathrm{a}$ \\
\hline Coating & Thyme $(0.1 \%)$ & 6.7 & 6.7 & 4.7 & 3.5 & 3.2 & $0.172 \mathrm{a}$ \\
\hline
\end{tabular}

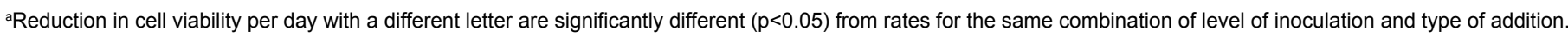

Table 7: Reduction in cell viability for Salmonella Typhimurium in extruded pet food products

the sensory properties of the product. The combination cinnamonspearmint $(21.14 \pm 4.37 \mathrm{~mm})$ showed a strong inhibitory effect against Salmonella, followed by cinnamon-thyme $(20.90 \pm 3.15 \mathrm{~mm})$ and cinnamon-eucalyptus $(19.83 \pm 1.36 \mathrm{~mm})$ (Table 4). However, none of them were statistically higher than the effect of cinnamon by itself $(20.66 \pm 4.45 \mathrm{~mm})$ on this microorganism.

For $P$. roqueforti, all the combinations that included cinnamon showed a strong inhibition (Table 4). All cinnamon combinations, with the exception of cinnamon-eucalyptus, showed significantly more inhibition than cinnamon essential oil by itself $(25.40 \pm 4.48$; data not shown; $\mathrm{p}<0.05)$. This effect can be explained by the presence of different antibacterial compounds, such as polyphenols in spices like cinnamon, and terpenes in herbs like thyme. A similar synergy was reported by Tunc et al. [30] when using cinnamaldehyde and carvacrol, the main components of cinnamon oil and thyme oil, to control the growth of Penicillium notatum.

Based on the previous results, cinnamon, thyme, spearmint, eucalyptus, peppermint, cinnamon-spearmint, cinnamon-thyme and cinnamon- peppermint were tested by an agar dilution method to determine the minimum inhibitory concentration against the microorganisms of interest. The essential oils used against Salmonella showed a MIC of $0.05 \%$ for cinnamon, cinnamon-spearmint and cinnamon-thyme; a MIC of $0.1 \%$ for thyme; and a MIC $>1.0 \%$ for spearmint. For $P$. roqueforti, the oils and combinations showed a MIC of $0.01 \%$ for cinnamon, cinnamon-spearmint and cinnamon-peppermint; a MIC of $0.5 \%$ for spearmint, a MIC of $1.0 \%$ for eucalyptus and MIC $>1.0 \%$ for peppermint. Contrary to our observations in the disk diffusion method, the addition of herbal EOs to cinnamon EO did not increase the antifungal activity in the agar dilution method. Comparing the MIC values found with published data is challenging, since there is no standardized method for the determination of antimicrobial potency [31]. In addition to the natural variability of essential oils, there are a large number of variations across studies on culture medium, size of inoculum, test method and measure of performance. For example, Cosentino et al. [32] found that the MIC of thyme essential oil for Salmonella Typhimurium ATCC 14028 was $0.9 \%$ for commercial oil and $0.45 \%$ for laboratory-distilled oil, using a broth microdilution 
method with Nutrient Broth (NB) as growth media. Pattnaik et al. [27] found MICs of eucalyptus and peppermint of $0.01 \%$ or lower against eleven fungi, but they did not test any Penicillium species. Though it is impossible to compare results across studies, it can be noted that the MICs determined by the method described here fall within the ranges described in the literature.

The oils that showed the best results to inhibit Salmonella were cinnamon $(\mathrm{MIC}=0.01 \%)$ and thyme $(\mathrm{MIC}=0.1 \%)$, while the best results against $P$. roqueforti were obtained with cinnamon $(\mathrm{MIC}=0.05 \%)$ and spearmint $(\mathrm{MIC}=0.5 \%)$. Therefore, these essential oils were chosen for the preparation of an extruded pet food product, with the oils as part of the product recipe or as part of the product coating. An additional level of $0.1 \%$ was included for cinnamon, to account for the possibility of lower activity in the product. As previously discussed, combinations were excluded as they did not present an improvement over the individual oils.

The average water activity of the products was 0.83 for uncoated product and 0.82 for coated products. Based on their water activity, these products can be classified as an Intermediate Moisture Food (IMF). The uncoated products with essential oil as part of the recipe showed no difference when the mold growth rate or the bacteria death rate were compared to those of the controls. The addition of oil into the product mix before extrusion may have allowed the antimicrobial compounds to vaporize, lowering their final concentration and effectiveness in the oil-in-product matrix. Most likely, it would be necessary to add a higher initial concentration of essential oil to observe the antimicrobial effect in this type of product. Holley notes that the final amounts of essential oils needed for activity in food systems might be as high as 1 to $3 \%$. Cost and palatability should be considered when adding essential oils to products, so some of these formulations perhaps would not be practical.

The only treatment that showed a significant difference in death rate of Salmonella from the control was the product coated with $0.1 \%$ cinnamon oil $(0.136 \log \mathrm{CFU} / \mathrm{g} /$ day for the control and 0.255 $\log \mathrm{CFU} / \mathrm{g} /$ day for the treatment, $\mathrm{p}=0.0431$ ), at the lower inoculation level. The final reduction achieved with this treatment was $4.4 \mathrm{log}$ $\mathrm{CFU} / \mathrm{g}$, with a $3 \log \mathrm{CFU} / \mathrm{g}$ reduction in the first 14 days. The addition of spice materials as part of dips, surface coatings or films for food systems has been reported to be effective before. These applications reduce the total amount of essential oil needed, while maintaining the required concentration of oil and ensuring its availability to interact with microorganisms. The greater activity shown by cinnamon in the final product compared to the other oils tested agrees with the results published by Burt, in which oregano, clove, coriander and cinnamon rank higher in antibacterial activity for different microorganisms in food systems. Thyme was classified as having a lower activity in this ranking, which is comparable to the present results.

The effect of cinnamon oil at $0.1 \%$ of the coating was not significant on the product inoculated with $107 \mathrm{CFU} / \mathrm{g}$. At this inoculation level, the death rates for Salmonella were similar for all treatments, including the control. The lack of effect can be attributed to the higher level of inoculation, as it is also slightly higher than the level tested while determining the MIC $\left(5 \times 10^{6} \mathrm{CFU} / \mathrm{g}\right)$. NACMCF [33] recommends using high numbers of organisms for inactivation studies such as this one, in order to quantify survivors or and/or to document high levels of inactivation. However, these numbers should also be consistent with the expected pathogen contamination levels (NACMCF, 2010).
It is known that Salmonella would not survive the extrusion conditions used for preparing the pet food product [34]. Therefore, the presence of Salmonella in extruded pet food products is usually due to postprocessing contamination in the processing plant. In these instances, usually the levels of Salmonella are generally low and the use of enrichment techniques for detection of the pathogen in the final product is required. Therefore, the performance of the cinnamon oil coating at $0.1 \%$ is considered satisfactory, since it would be used strictly to manage post-processing contamination.

When the different methods used here to determine antimicrobial activity of essential oils are compared, Salmonella inhibition was achieved in the agar dilution method with $0.05 \%$ of cinnamon essential oil, while $0.1 \%$ of cinnamon essential oil was required to obtain a significant effect in the extruded product. On the other hand, $P$. roqueforti was inhibited in vitro with less cinnamon essential oil (0.01\%), but no effect was observed in the extruded product, even at an addition level of $0.1 \%$. The decreased antimicrobial activity found in the food product compared to the in vitro assay has been described before and may be explained by the complexity of food matrixes and their processing. Food systems usually have greater availability of nutrients than laboratory media and some of its components, such as moisture, protein and fat, might have protective effects for bacteria. For example, Smith-Palmer et al. [35] found that cinnamon oil at $0.075 \%$ showed antimicrobial activity against L. monocytogenes in TSB, but the amount had to be raised to $1 \%(\mathrm{v} / \mathrm{v})$ to observe activity in full fat cheese. Essential oils are mostly hydrophobic and can be easily dissolved in food lipids, which means a decreased availability in the hydrophilic portion where bacteria are located. In addition, $\mathrm{pH}$ may enhance the hydrophobic quality of these compounds.

Additionally, antimicrobial potency is also usually reduced in products with lower water activity. Based on the water activity levels achieved ( 0.83 for uncoated product and 0.82 for coated products), the product can be classified as an Intermediate Moisture Food (IMF), which is characterized by water activity of $0.65-0.90$. P. roqueforti growth is common in IMF [36] and unfortunately, the essential oil concentrations used in the present study were not sufficient to control the growth of $P$. roqueforti since the water activity of the pet food product supported its growth. Salmonella, on the other hand, requires water activity above 0.90 for growth, so its reduction over time in all products was expected [37,38]. However, the reduction of Salmonella brought on by the disadvantageous water activity of the product was further enabled by the presence of cinnamon essential oil at $0.1 \%$ in the coating. The results obtained are aligned with the concept of multiple hurdles, which states that a combination of several factors can be effectively used to control microorganisms [39].

\section{Conclusions}

Different plant essential oils had in vitro inhibitory activity against Salmonella Typhimurium and Penicillium roqueforti. Inhibitory activity was dependent on the type and concentration of essential oil. Cinnamon essential oil showed high inhibitory activity against Salmonella and $P$. roqueforti in vitro, with a minimum inhibitory concentration of $0.05 \% \mathrm{v} / \mathrm{v}$ in agar for both organisms. When applied as part of the coating of a pet food product, $0.1 \%$ cinnamon essential oil had no effect on $P$. roqueforti growth, but lowered the survival population of Salmonella. Based on the results, spice essential oils can act as inhibitors of Salmonella in a pet food product, when present at a sufficient concentration. 
Citation: Bianchini A, Stratton J, Weier S, Cano C, Garcia LM, et al. (2014) Use of Essential Oils and Plant Extracts to Control Microbial Contamination in Pet Food Products. J Food Process Technol 5: 357. doi:10.4172/2157-7110.1000357

\section{Acknowledgments}

This work was supported by the Layman Award provided by the Office of Research and Economic Development at the University of Nebraska-Lincoln.

\section{References:}

1. Centers for Disease Control and Prevention (CDC) (2006) Human salmonellosis associated with animal-derived pet treats--United States and Canada, 2005. Morbidity and Mortality Weekly Report 55: 702-705.

2. Centers for Disease Control and Prevention (CDC) (2008) Update: recall of dry dog and cat food products associated with human Salmonella Schwarzengrund infections-United States, 2008. Morbidity and Mortality Weekly Report 57: $1200-1202$.

3. Centers for Disease Control and Prevention (CDC) (2012) Notes from the field: Human Salmonella infantis infections linked to dry dog food--United States and Canada, 2012. MMWR Morb Mortal Wkly Rep 61: 436.

4. Clark C1, Cunningham J, Ahmed R, Woodward D, Fonseca K, et al. (2001) Characterization of Salmonella associated with pig ear dog treats in Canada. J Clin Microbiol 39: 3962-3968.

5. Pitout JD1, Reisbig MD, Mulvey M, Chui L, Louie M, et al. (2003) Association between handling of pet treats and infection with Salmonella enterica serotype newport expressing the AmpC beta-lactamase, CMY-2. J Clin Microbiol 41: 4578-4582.

6. Crane SW, Griffin RW, Messent PR (2000) Introduction to commercial pet foods), Small Animal Clinical Nutrition Marceline. (4thedn). MO: Walsworth Publishing Company.

7. Hammer KA, Carson CF, Riley TV (1999) Antimicrobial activity of essential oils and other plant extracts. J Appl Microbiol 86: 985-990.

8. Smith-Palmer A, Stewart J, Fyfe L (1998) Antimicrobial properties of plant essential oils and essences against five important food-borne pathogens. Lett Appl Microbiol 26: 118-122.

9. Azzouz M, Bullerman L (1982) Comparative antimycotic effects of selected herbs, spices, plant components and commercial antifungal agents. Journal of Food Protection 45: 1298-1301.

10. Paster N, Menasherov M, Ravid U, Juven B (1995) Antifungal activity of oregano and thyme essential oils applied as fumigants against fungi attacking stored grain. Journal of Food Protection 58: 81-85.

11. Careaga M, Fernández E, Dorantes L, Mota L, Jaramillo ME, et al. (2003) Antibacterial activity of Capsicum extract against Salmonella typhimurium and Pseudomonas aeruginosa inoculated in raw beef meat. Int $J$ Food Microbiol 83: 331-335

12. Koutsoumanis K, Lambropoulou K, Nychas GJ (1999) A predictive model for the non-thermal inactivation of Salmonella enteritidis in a food model system supplemented with a natural antimicrobial. International journal of food microbiology 49: 63-74

13. Tassou C, Drosinos E, Nychas G (1995) Effects of essential oil from mint (Mentha piperita) on Salmonella enteritidis and Listeria monocytogenes in model food systems at 4 degrees and 10 degrees C. J Appl Bacteriol 78: 593-600.

14. Baker D, McGenity P (2003) Animal food composition. United States.

15. Qvyjt F (2005) Pet food composition having enhanced palatability. WO Patent $2,005,053,424$. United States.

16. Sunvold GD, Corrigan PJ (2010) Pet food having improved animal preference. United States.

17. KritzmanA, MarkusA, Strongin P, Linder C (2009)Applications of microencapsulated essential oils. United States.

18. Nahas R, Chadwick L, Berdahl D, McKeague J, Vanalstyne CP, et al. (2010) Compositions and methods for enhancing the stability of foods, beverages, nutritional supplements and cosmetics. United States.
19. Boysen ME, Jacobsson KG, Schnürer J (2000) Molecular identification of species from the Penicillium roqueforti group associated with spoiled animal feed. Appl Environ Microbiol 66: 1523-1526.

20. Centers for Disease Control and Prevention (CDC) (2013) FoodNet-Tables and Figures 2012 Preliminary Data.

21. Packiyasothy EV, Kyle S (2002) Antimicrobial properties of some herb essentia oils. Food Australia 54:384-387.

22. Holley RA, Patel D (2005) Improvement in shelf-life and safety of perishable foods by plant essential oils and smoke antimicrobials. Food Microbiol 22: 273-292.

23. Davidson PM, Naidu AS (2000) Phyto-Phenols. Natural Food Antimicrobial Systems Boca Raton, FL: CRC Press.

24. Suhr KI, Nielsen PV (2003) Antifungal activity of essential oils evaluated by two different application techniques against rye bread spoilage fungi. J Appl Microbiol 94: 665-674.

25. Prabuseenivasan S, Jayakumar M, Ignacimuthu S (2006) In vitro antibacteria activity of some plant essential oils. BMC Complement Altern Med 6: 39

26. Alfazairy A (2004) Antimicrobial activity of certain essential oils against hindgut symbionts of the drywood termite Kalotermes flavicollis Fabr. and prevalent fungi on termite-infested. Journal of Applied Entomology 128: 554-560.

27. Pattnaik S, Subramanyam VR, Kole C (1996) Antibacterial and antifungal activity of ten essential oils in vitro. Microbios 86: 237-246.

28. Roldan LP, Diaz GJ, Duringer JM (2010) Composition and antibacterial activity of essential oils obtained from plants of the Lamiaceae family against pathogenic and beneficial bacteria. Revista Colombiana de Ciencias Pecuarias 23: $451-461$

29. Delaquis PJ, Stanich K, Girard B, Mazza G (2002) Antimicrobial activity of individual and mixed fractions of dill, cilantro, coriander and eucalyptus essential oils. International Journal of Food Microbiology 74: 101-109.

30. Tunc S1, Chollet E, Chalier P, Preziosi-Belloy L, Gontard N (2007) Combined effect of volatile antimicrobial agents on the growth of Penicillium notatum. Int $J$ Food Microbiol 113: 263-270.

31. Burt S (2004) Essential oils: their antibacterial properties and potential applications in foods--a review. Int J Food Microbiol 94: 223-253.

32. Cosentino S, Tuberoso Cl, Pisano B, Satta M, Mascia V, et al. (1999) In-vitro antimicrobial activity and chemical composition of Sardinian Thymus essential oils. Lett Appl Microbiol 29: 130-135.

33. NACMCF (2010) Parameters for determining inoculated pack/challenge study protocols. J Food Prot 73: 140-202.

34. Bianchini A, Stratton J, Weier S, Hartter T, Plattner B, et al. (2014) Use of Enterococcus faecium as a surrogate for Salmonella enterica during extrusion of a balanced carbohydrate-protein meal. J Food Prot 77: 75-82.

35. Smith-Palmer A, Stewart J, Fyfe L (2001) The potential application of plant essential oils as natural food preservatives in soft cheese. Food Microbiol 18 463-470.

36. Matan N, Rimkeeree H, Mawson AJ, Chompreeda P, Haruthaithanasan V et al. (2006) Antimicrobial activity of cinnamon and clove oils under modified atmosphere conditions. Int J Food Microbiol 107: 180-185.

37. Himathongkham S, Nuanualsuwan S, Riemann H (1999) Survival of Salmonella enteritidis and Salmonella typhimurium in chicken manure at different levels of water activity. FEMS Microbiol Lett 172: 159-163.

38. Koutsoumanis K, Kendall PA, Sofos JN (2004) Modeling the boundaries of growth of Salmonella typhimurium in broth as a function of temperature, water activity and pH. J Food Prot 67: 53-59.

39. Leistner L, Gorris LGM (1995) Food preservation by hurdle technology. Trends in Food Science \& Technology 6: 41-46. 